\title{
Gewalt als die niedrigste Ebene der Konfliktlösung
}

\section{Georg Lind (Konstanz)}

Zur Natur der Gewalt gibt es zwei grundsätzlich verschiedene, information pills teilweise völlig entgegengesetzte Vorstellungen, sildenafil die ihrerseits unsere Bemühungen um Verringerung von Gewalt beeinflussen: Auf der einen Seite die Vorstellung, dass Gewalt eine eigenständige menschliche Eigenschaft sei, die ihre eigene Entwicklungsgeschichte hat, und die direkt bekämpft oder eingedämmt werden muss, sei es wiederum durch Mittel der Gewalt oder durch ?trickreiche? Methoden der dosierten Abfuhr oder der Ablenkung auf vermeintlich gefahrlosere Gebiete wie sportlicher oder ökonomischer Wettkampf.

Auf der anderen Seite gibt es die Vorstellung, dass Gewalt eine von vielen Arten der Konfliktlösung ist, und zwar die niedrigste Form oder Ebene, die Menschen dann ?wählen?, wenn ihnen - subjektiv - keine adäquatere Form der Konfliktlösung zur Verfügung steht. Nach dieser Vorstellung kann Gewalt nicht ?bekämpft? werden, sondern kann nur dadurch verhindert werden, dass höher entwickelte, rationale Formen der Konfliktlösung gefördert werden.

Beide Vorstellungen werden in diesem Vortrag auf der Grundlage empirischer Studien gegenübergestellt. Der zentrale Befund ist, dass (politisch motivierte) Gewaltneigung mit einer geringen moralischen Urteilsfähigkeit korreliert - eine Bestätigung der These, dass Gewalt Anzeichen einer geringen Problemlösekompetenz ist. Mit hoher moralischer Urteilsfähigkeit ist in der Regel ein reflektiertes Engagement verbunden, das demokratisch und sich dem Allgemeinwohl verpflichtet fühlt.

\section{Einleitung}

Die zentrale These meines Vortrags ist, dass Gewalt eine Form von Konfliktlösefähigkeit darstellt, genauer: die niedrigste Fähigkeit, die Menschen zur Verfügung steht, und dass sie daher am besten eingedämmt oder vermieden werden kann, indem man Menschen befähigt, höhere, bessere Formen der Konfliktlösung zu wählen oder zu entwickeln.

Beide Teile dieser These gehören eng zusammen. Diese These kontrastiert mit der Ansicht, dass Gewalt eine Grundmotivation darstellt, die Menschen befriedigen müssen, und der damit korrelierten Ansicht, dass man Gewalt am besten eindämme, indem man sie ?bekämpft?. Auch diese beiden Teile gehören zusammen.

Schauen wir uns zunächst die einzelnen Bestandteile meiner Thesen genauer an, bevor wir daran gehen, empirische Anhaltspunkte zu diskutieren, die sie unterstützen bzw. in Frage stellen. Es geht um zwei Kernbegriffe, nämlich um ?Gewalt? und um ?Fähigkeit?.

\section{Was ist ?Gewalt??}

Obwohl schon viele Versuche der Definition von Gewalt unternommen wurden, Gewalt zu definieren, gehen viele dieser Versuche an den eigentlichen Merkmalen vorbei. Frech (1993) definiert Gewalt als ein zielgerichtetes Schädigen und Beeinträchtigen anderer Menschen” (S. 60). Gewalt wird häufig hier synonym mit Aggression gesetzt. So definiert Nolting (1993) auch Aggression als „ein zielgerichtetes oder zumindest ?gerichtetes? Schädigen, Beeinträchtigen und Schmerzzufügen (z.B. andere Menschen verletzen, töten, bedrohen, beschimpfen, herabsetzen)? (S. 91; siehe auch Petermann \& Petermann, 1984; Willems, 1994). Einige fassen Gewalt weiter und bezeichnen so auch jede psychologische oder sozio-strukturelle Schädigung. Gewalt, so Galtung (1993), bezeichnet die ?vermeidbare Beeinträchtigung grundlegender menschlicher Bedürfnisse oder, allgemeiner ausgedrückt, des Lebens, die den realen Grad der Bedürfnisbefriedigung unter das herabsetzt, was potentiell möglich ist? (S. 106).

Obwohl all diese Definitionen ungefähr das treffen, was wir meinen, wenn wir von Gewalt sprechen, überbetonen sie ein Merkmal, nämlich die Zielgerichtetheit, und übersehen dafür ein anderen gänzlich, nämlich die Frage der Gerechtigkeit oder Adäquatheit. Das Kriterium Zielgerichtetheit schließt aus, dass das Verhalten einer Person, die jemanden anders zufällig schädigt, als gewalttätig bezeichnet wird. Zum Beispiel: ein Kind fuchtelt aus Spaß mit den Armen in der Luft herum und schlägt dabei zufällig ein anderes Kind an den Kopf. Ein solches Verhalten bezeichnen wir nicht als Gewalt gegen das andere Kind.

Was aber, wenn ein Kind bewusst ein anderes Kind schlägt? Sicherlich kann man es dann als gewalttätig bezeichnen. Aber liegt in einem solchen Fall immer eine gezielte Schädigung vor? Können wir dann immer davon ausgehen, dass das Kind motiviert war, das andere Kind zu schädigen, zu verletzen oder herabzusetzen? Oder kann es auch anderen Motive geben als eine GewaltMotivation?

Die Antwort ist ja, wie sich an Beispielen zeigen lässt. Nehmen wir an, ein Kind möchte aus irgendeinem für es wichtigen Grund zu einem Ziel gelangen, zu dem es nur einen engen Weg gibt. Ein anderes Kind stellt sich ihm in den Weg und weicht auch nicht, nachdem das erste Kind es inständig bittet. Da sieht das Kind keine andere Möglichkeit, als das Kind zu Boden zu schlagen, damit es zu seinem Ziel kommt. Das ?Opfer? wurde stark verletzt. Es entstand Schaden. Aber es lag eindeutig keine Absicht vor, 
dem anderen Kind zu schaden. Die Absicht war allein, das Ziel zu erreichen. Dennoch nennen wir dieses Verhalten zu recht gewalttätig.

Es kann also Gewalt geben, ohne dass das Verhalten des Handelnden auf die Schädigung, Verletzung oder Tötung eines anderen Menschen zielt oder durch solche Ziele motiviert ist. Ich würde sogar behaupten, dass die meisten Fälle von gewalttätigem Verhalten zu dieser Kategorie gehören. Zwar sind wir im Zustand der Erregung über solche Taten gern geneigt anzunehmen, dass die Tat ?böswillig? und mit voller Schädigungsabsicht erfolgt ist. Bei der genauen, vorurteilsfreien Durchsicht vieler Fälle wird man aber zu dem Schluss kommen, dass es nur ganz wenige Gewalthandlungen gibt, hinter denen sich eine primäre Schädigungsabsicht verbirgt. Es kommt sicher häufig vor, dass die Schädigung einer anderen Person billigend in Kauf genommen wird, wenn ein bestimmtes Ziel nicht anders zu erreichen ist, aber als primäres Handlungsziel spielt, so lese ich die Forschungsliteratur, in den seltensten Fälle eine Rolle.

Dies ist eine Unzulänglichkeit der meisten Definitionen von Gewalt. Würde man darauf bestehen, dass nur Handlungen als gewalttätig bezeichnet werden können, die Gewalt zum Ziel haben, dann gäbe es kaum Gewalt. Das widerspräche aber unserem Erleben. Bezeichnet man aber alles Verhalten, das Gewalt billigend in Kauf nimmt, oder das als Mittel zur Erreichung von übergeordneten Zielen oder der Befriedung anderer Bedürfnisse geschieht, entsteht die Schwierigkeit, dass plötzlich auch solches Verhalten als Gewalt erscheint, das wir grundsätzlich bejahen: zum Beispiel die Arbeit eines Chirurgen, der unseren Körper verletzt und das Risiko der Tötung in Kauf nimmt, um uns von einer schweren Krankheit zu befreien. Oder ein Mensch, der in Notwehr einen anderen tötet. Noch mehr Beispiele sind zu finden, wenn man den Begriff der Gewalt ausweitet auf die strukturelle oder gar die kulturelle Gewalt (Galtung, 1993).

Ein aktuelles Beispiel ist die gegenwärtige Sparpolitik in vielen Ländern der Europäischen Union. Um den langfristigen Wohlstand in diesen Ländern zu sichern, muss man die ausufernden Schulden in den Griff bekommen. Manche Politiker sehen dabei keinen anderen Weg, als die ärmeren Teile der Bevölkerung noch ärmer zu machen (Der Spiegel Nr. 40, 1997; Martin \& Schumann, 1997). Galtung spricht hier von ?strukturelle Gewalt?. Wir konzedieren, dass diese Form der Gewalt nicht das eigentliche Ziel von denjenigen ist, die über Lohnkürzungen für gering Verdienende beschließen. Sie wird aber von ihnen in Kauf genommen.

\section{Gewalt als mangelnde Problemlösefähigkeit}

Wie können wir diese Diskrepanz zwischen der gängigen Definition von Gewalt und obigen Phänomenen besser miteinander in Einklang bringen? Eine Möglichkeit wäre zu sagen, unser Empfinden ist ungenau oder gar falsch und wir müssen versuchen, es den offenbar weithin akzeptierten Definitionen anpassen. Ich glaube nicht nur, dass eine solche Bemühung zum Scheitern verurteilt ist, sondern auch, dass sie falsch ist. Sie verkennt, dass auch eine Definition falsch sein kann, weil sie einen sozialen oder psychologischen Tatbestand nur unzureichend wiedergibt, ein nicht seltener Fall in den Sozialwissenschaften. Sie verkennt auch, dass häufige Zitation nicht unbedingt ein Zeichen für eine reflektierte Akzeptanz sein muss, sondern vielmehr Denkfaulheit und Konformität entspringen kann. Auch dies kein seltenes Phänomen.

Wir müssen daher genauer hinzusehen, warum wir ein bestimmtes Verhalten als gewalttätig bezeichnen und anderes nicht. Bei solchem genaueren Hinsehen stellt man fest, dass es auf die Art der Mittel ankommt, mit denen jemand seine Ziele zu erreichen versucht, ob man ein Verhalten als - ?Gewalt? bezeichnet oder nicht. Zum Beispiel das Ziel Sexualverkehr haben, wird gemeinhin nicht als ein Gewaltakt bezeichnet. Gewalttätig nennen wir es nur, wenn dieses Ziel zu erreichen versucht wird, ohne dass das Einverständnis des Sexualpartners vorliegt. Dieses Einverständnis zu erreichen, ist oft an Bedingungen geknüpft, die für manchen leicht, für andere schwer zu erreichen sind. Solche Bedin- gungen sind oft nicht einmal äußere Attribute wie Jugend, Schönheit und Reichtum, sondern die Fähigkeit der sozialen Kontaktaufnahme, Vertrauenswürdigkeit, Achtung vor den Bedürfnissen der anderen Person und ähnliches mehr, die es zu erfüllen gilt, um das Ziel (das Einverständnis der anderen Person) zu erreichen. Allgemein formuliert können wir sagen: wir empfinden jenes Verhalten als Gewalt, das ein Ziel unfair verfolgt, das heißt ohne Rücksicht auf andere Ziele oder die Ziele anderer. Gewalt hat also mit Fragen der Gerechtigkeit und Moral zu tun. Wir empfinden ein Verhalten umso gewalttätiger, je weniger es sich um Fragen der Gerechtigkeit und Moral kümmert. Werbik und Kempf sprechen daher von Gewalt nur dann, wenn ein Handeln gegen grundlegende Rechte oder einen allgemein anerkannten Bedarf eines anderen verstößt (Kempf, 1983, S. 22).

Hier handelt es sich also nicht bloss um eine neue Definition unter vielen, sondern um ein neues Verständnis von Gewalt mit weitreichenden Konsequenzen für die Erklärung ihrer Entstehung und für die Mittel zur ?Bekämpfung? oder ?Überwindung? von Gewalt. Bei den beiden Wörtern zeigt sich schon ein tiefer Unterschied. ?Bekämpfen? wird man Gewalt sinnvollerweise nur dann, wenn man glaubt, die Gewalt sei eine psychische Kraft, Motivation oder Trieb. Nimmt man hingegen an, sie sei eigentlich ein Negativ-Phänomen, nämlich die Abwesenheit von adäquateren Problemlösungen, wird ein ?Bekämpfen? sinnvoll. In diesem Fall ist eher die Ausbildung solcher Problemlösefähigkeiten angesagt, um Gewalt sozusagen überflüssig zu machen. „Wachsende soziale Kompetenz?, so Krappmann und Oswald (1995), ?macht den Rückgriff auf Gewalt obsolet? (S. 136). Nach diesem Verständnis kommt es zu Gewalt nur dann, wenn die Problemlösekompetenz gering ist.

Ich will noch einen Schritt weitergehen und sagen, Gewalt definieren als die niedrigste Form oder Stufe der individuellen Problemlösekompetenz. Mit dieser Annahme eines einzigen Konstrukts können wir die gleichen Phänomene beschreiben wie 
mit Annahme, dass hier zwei verschiedene, negativ korrelierte Konstrukte vorliegen, und sie ist sparsamer, was für wissenschaftliche Theorien immer vorteilhaft ist, wenn man sie in der Praxis anwenden will. Bei gleicher Aussagekraft ist die sparsamere Theorie immer auch diejenige, die leichter lehrbar und in der Praxis anwendbar ist.

Wenn man die niedrigste Stufe der Problemlösekompetenz als jene bezeichnet, bei der Gewalt zur Lösung von Konflikten herangezogen wird, dann könnte man in Anlehnung an Krappmann und Oswald (1995) und Habermas (1983) als die höchste Stufe diejenige bezeichnen, bei der Konflikte auf der Basis gemeinsamer, universeller Moralprinzipien zu lösen versucht werden. „Moralisches Handeln steht unter dem Anspruch,? so Habermas (1983), ?dass sich die Beilegung von Handlungskonflikten allein auf begründete Urteile stützt - es ist ein durch moralische Einsichten geleitetes Handeln? (S. 174). Somit schließt ?die moralische Lösung von Handlungskonflikten schließt die manifeste Anwendung von Gewalt ebenso aus wie einen billigen Kompromiss; sie lässt sich als eine Fortsetzung kommunikativen, d.h. verständigungsorientierten Handelns mit diskursiven Mitteln verstehen? (S. 74). Ähnlich definiert auch Kohlberg eine entwickelte moralische Urteilsfähigkeit als die Überwindung jener Entwicklungsstufen, auf denen Gewalt zur Lösung sozio-moralischer Konflikte angewendet wird (Kohlberg, 1995, S. 336 ff.). Unter Heranziehung von Kohlbergs Stufenmodell der Moralentwicklung lässt sich lässt sich eine Entwicklungstheorie der Problemlösekompetenz wie in Abbildung 1 skizzieren.

\section{Methoden}

Zur Untermauerung meiner These will ich hier über einschlägige, bislang unveröffentlichte Befunde aus einer Untersuchung bei Abiturienten aus dem Jahr 1978 berichten (Peisert, Hg., 1981). Untersucht wurde ein repräsentatives Sample von 708 Schülern und Schülerinnen der 13. Klasse in Baden- Württemberg. Für die Prüfung unserer Hypothese spielt der Zeitpunkt der Untersuchung keine grosse Rolle.

Die für unsere Fragestellung hier wichtigen Variablen Problemlösefähigkeit und Gewaltneigung wurden mit zwei Instrumenten erfasst, mit dem Moralisches-Urteil-Test (MUT) von Lind $(1978 ; 1984,1995)$ und der New-Left-Skala von Christie und Geis (Robinsohn \& Shaver, 1973).

\section{Moralisches-Urteil-Test (MUT)}

Der MUT erfasst neben moralischen Einstellungen auch moralische Urteilsfähigkeit. Damit ist die Fähigkeit gemeint, sich bei der Lösung von Konflikten an moralischen Prinzipien zu orientieren. Anders als bei Kohlberg, der als Index für diese Fähigkeit die Häufigkeit auswertet, mit der bestimmte Stufen des moralischen Urteilens bei der Lösung eines Problems benutzt werden, wird diese Fähigkeit beim MUT dadurch gemessen, wie konsistent sich eine Person an den selbstgewählten Maßstäben orientiert, unabhängig davon, um welchen Maßstab es sich hierbei handelt.

Es wird dabei nicht irgendeine Urteilskonsistenz herangezogen, sondern die Konsistenz des Urteilsverhaltens der Befragten in Bezug auf moralische Orientierungen (unterschiedlicher Qualität) unter erschwerten Bedingungen. Die Schwere der Aufgabe für die Befragten besteht darin, sowohl Argument zu beurteilen, die für die Lösung sprechen, die sie wählen, sondern auch Argumente, die gegen die präferierte Lösung sprechen.

Die Vermutung, dass es sich hierbei wirklich um eine schwere Aufgabe handelt, konnten durch verschiedene Untersuchungen bestätigt werden. In zwei Laborexperimenten (Lind, 1993; Wasel,1993) hat sich gezeigt, dass Versuchspersonen die Aufgabe nicht einfach dadurch lösen konnten, dass sie dazu aufgefordert wurden. Die dafür benötigte Urteilsfähigkeit erwies sich als nicht simulierbar. Offenbar stellt die sachliche Auseinandersetzung mit Gegenargumenten eine hohe Entwicklungsstufe der Urteilsfähigkeit dar.

In der Standard-Version des MUT, die wir auch hier verwendeten, werden den Befragten zwei Dilemmas in Form von kurzen Geschichten vorgelegt (siehe Anhang). Sie werden gebeten, zunächst ihre Meinung zu der Lösung anzugeben, die in der Geschichte gewählt wurde (Frage zum Sterbehilfe- Dilemma: ?Beurteilen Sie das Verhalten des Arztes eher als falsch oder eher als richtig??). Die Antwort wird durch Ankreuzen auf einer Skala von -3 bis +3 gegeben. Anschließend an diese ?Meinungsfrage? müssen die Befragten jeweils sechs Argumente für und sechs Argumente gegen die vorgelegte Dilemmalösung beurteilen (?Wie akzeptable sind folgende Argument...??) Auch hier wieder können die Antworten auf einer Skala angekreuzt werden, diesmal von -4 (?völlig unakzeptabel?) bis +4 (?völlig akzeptabel?).

Aus den Antworten der Befragten wird der C-Wert (C für competence) gebildet. Der C-Wert gibt an, zu welchem Grad eine Person fähig ist, Pro- und Kontra-Argumente konsistent in Bezug auf moralische Prinzipien zu beurteilen, statt sie zum Beispiel danach zu beurteilen, wie weit sie mit der eigenen Meinung übereinstimmen. Er reicht von ?o" (keine Fähigkeit, sich konsistent an moralischen Prinzipien zu orientieren) bis 100. Für technisch interessierte: Der C-Wert wird mittels einer intra- individuellen multivariaten Varianzkomponenten-Zerlegung berechnet. Dies wird durch das Design des MUT als $N=1$-Experiment mit einem dreifaktoriellen, orthogonalen Design nahegelegt.

\section{New Left-Skala (NL)}


Die hier verwendete Version der New-Left Skala von Christie und Geis beinhaltet zwanzig Fragen, die sich konzeptionelle und empirisch zwei Dimensionen zuordnen lassen, a) der Dimension ?Philo- sophie der Neuen Linken? (der 68er Generation) und b) der Dimension ?Revolutionär- gewaltorientierte Taktiken?. Die Dimensionen ?Philosophie der Neuen Linken? beschreibt also die politischen Zielsetzungen. Sie ist in der Skala durch Sätze repräsentiert wie: (-) ??Demokratisierung aller Lebensbereiche sollte die Grundlage einer neuen Gesellschaft sein.? (+). Die Dimension revolutionär-gwaltorientierte Taktiken? beschreibt dagegen die Mittel zur Durchsetzung dieser Ziele. Sie ist durch Sätze räpresentiert wie:

- ?Unsere Gesellschaft kann eher durch gewaltsame Störungen als durch Diskussionen verändert werden.? (+)

- $\quad$ ?Es gibt legitime Wege der Reform, die zuerst ausgeschöpft werden müssen, bevor man zu gewaltsamen Mitteln greift.? (-)

Die Antwortmöglichkeiten bei der NL-Skala reichen von ?1" (lehne stark ab) bis ?7" (stimme stark zu).

\section{Befunde}

Unsere Untersuchung bei den Abiturienten hat unsere These bestätigt. Zwischen der Neigung zu Gewalt und Problemlösefähigkeit (hier operationalisiert als moralische Urteilsfähigkeit) besteht tatsächlich ein systematischer Zusammenhang (Abbildung 2). Bei jenen, die der Überzeugung sind, Änderungen der Gesellschaft können nur durch gewaltsame Störungen erreicht werden, liegt der Messwert für moralische Urteilsfähigkeit (C-Wert) deutlich tiefer $(\mathrm{M}=30,2)$ als bei denen, die sich unsicher sind $(M=36,0)$ und denen, die Gewalt deutlich ablehnen $(M=40,7)$. Die Differenz zwischen den Extremgruppen beträgt über 10 Skalenpunkte bei einer Gesamtlänge der Skala von 10o Punkten. Das ist, wie der Vergleich mit anderen Untersuchungen zeigt (Lind, 1993), eine sehr bedeutsame Differenz. Der Unterschied wäre vermutlich größer ausgefallen, wenn man wir eine heterogenere Untersuchungsgruppe gehabt hätten. Bei unseren Abiturienten war die Antwortvarianz relativ gering. ${ }^{1}$

Die Korrelation des C-Wertes mit der gesamten Unterskala ?Revolutionär-gewaltorientierte Taktiken? beträgt in dieser Gruppe von Abiturienten $r=-0.20$. Das heißt, je höher die moralische Urteilsfähigkeit, desto geringer ist die Neigung der Befragten, gewaltorientierten Mitteln zur Durchsetzung politischer Zielsetzungen zuzustimmen.

Eine detailliere Analyse der Beziehung zwischen Gewaltneigung auf der einen Seite und der Einstellung der Befragten zu den sechs von Kohlberg beschriebenen Stufen der Moralorientierung bestätigen diesen Befund (Abbildung 3).

Völlig gegenläufig ist dagegen die Beziehung zwischen der zweiten Dimension der New-Left-Skala, den (linken) politischen Zielsetzungen und der moralischen Urteilsfähigkeit (Abbildung 4). Wer der Forderung nach ?Demokratisierung aller Lebensbereiche? zustimmt, hat einen hohen mittleren C-Wert $(M=41,2)$, wer sie ablehnt dagegen einen im Mittel sehr viel niedrigeren ( $M=26,5)$. (Abbildungen siehe Anhang).

\section{Diskussion}

Die vorliegende Untersuchung von 708 Abiturienten hat einen negativen Zusammenhang zwischen moralischer Urteilsfähigkeit und Gewaltneigung, aber einen positiven Zusammenhang mit demokratischen politischen Zielsetzungen erbracht. Damit wird die These erhärtet, dass Gewaltneigung eine niedrige Form der Problemlösefähigkeit darstellt. Wenn eine Gewaltneigung vorliegt, dann steht dies bei vielen in Zusammenhang mit einer niedrigen moralischen Urteilskompetenz. Je höher dagegen die Fähigkeit ist, Konflikte auf der Basis moralischer Prinzipien zu lösen, desto mehr erscheint Gewalt als Mittel der politischen Auseinandersetzung ?obsolet?

Natürlich kann eine solche Korrelationsstudie keine Gewissheit über den vermuteten Kausalzusammenhang verschaffen. Aber sie ist geeignet die zentrale These zu testen. Durch eine positive oder sehr niedrige Korrelation wäre sie in Frage gestellt oder gar widerlegt worden. Auch aus anderen Studien liegen bestätigende Hinweise vor. So haben wir in einer großangelegten Interventionsstudie in drei Schulen in Nordrhein-Westfalen gefunden, dass unser Programm zur Förderung der moralischen Urteilsfähigkeit in der Schule zu einer Verringerung von Vandalismus und Gewalt unter Schülern geführt hat (Lind, 1993). Dennoch sind weitere Studien notwendig, diese These zu erhärten und die sich hieraus ergebenden Implikationen für eine effektive Konflikt- und Friedenspädagogik zu überprüfen.

\section{Literatur}

Frech, S. (1993). Aggression und Gewalt in der Schule. Bildung \& Wissenschaft 1993, S. 59?64.

Galtung, J. (1993). Kulturelle Gewalt. Der Bürger im Staat 43, Landeszentrale für politische Bildung Baden-Württemberg, S. $106 ? 112$.

Habermas, J. (1983). Moralbewusstsein und kommunikatives Handeln. Frankfurt/M.: Suhrkamp. 
Kempf, W. (1983). Aggression. In: E. Lippert \& R. Wakenhut (Hg.), Handwörterbuch der Politischen Psychologie. Opladen: Westdeutscher Verlag, S. 19?26.

Krappmann, L., \& Oswald, H. (1995). Alltag der Schulkinder: Beobachtungen und Analysen von Interaktionen und Sozialbeziehungen. Weinheim \& München: Juventa Verlag.

Lind, G. (1978). Wie mißt man moralisches Urteil? Probleme und alternative Möglichkeiten der Messung eines komplexen Konstrukts. In: G. Portele (Hg.), Sozialisation und Moral. Weinheim: Beltz, S. 171?201.

Lind, G. (1984). Theorie und Validität des Moralisches-Urteil-Tests. Zur Erfassung kognitiv struktureller Effekte der Sozialisation. In: G. Framhein \& J. Langer (Mithg.), Student und Studium im interkulturellen Vergleich. Klagenfurt: Kärtner Druck- und Verlagsanstalt, S. 166?187.

Lind, G. (1993). Moral und Bildung. Zur Kritik von Kohlbergs Theorie der moralisch-kognitiven Entwicklung. Heidelberg: Ansager.

Lind, G. (1995). The meaning and measurement of moral competence revisited - A dual aspect model. Eingeladener Vortrag, Jahrestagung der American Educational Research Association. San Francisco, 1995.

Martin, H.-P. \& Schumann (1997). Die Globalisierungsfalle. Reinbeck: Rewohlt.

Nolting, H.-P. (1993). Aggression ist nicht gleich Aggression. Ein Überblick aus psychologischer Sicht. Der Bürger im Staat 43. Landeszentrale für politische Bildung Baden-Württemberg, S. 91?95.

Robinson, J.P. \& Shaver, Ph.R. (1973). Measures of social psychological attitudes. Ann Arbor, MI: Survey Research Center.

Peisert, H. (Hg.), (1981). Abiturienten und Ausbildungswahl. Weinheim: Beltz.

Petermann, F. \& Petermann, U. (1984). Training mit aggressiven Kindern. München: Urban \& Schwarzenberg.

Shaver, J.P. (1993). What statistical significance testing is, and what it is not. Journal of Experimental Education, 61, S. 293 ?316.

Willems, H. (1994). Gewalt ? Ein Phänomen unserer Gesellschaft? Eine Bestandsaufnahme. In: B. Ebbert \& P. Wittemann (Hg.), ...und bist Du nicht willig... Gewalt ? Kinder und Jugendliche als Opfer und Täter, Stuttgart: Aktion Jugendschutz Landesarbeitsstelle Baden-Württemberg, S. 11?33.

Wasel, W. (1993). Simulation moralischer Urteilsfähigkeit. Moralentwicklung: eine kognitiv-strukturelle Veränderung oder ein affektives Phänomen?, Konstanz: unveröffentlichte Diplomarbeit, Universität Konstanz.

\section{Abbildungen}

Stufe 1: Einsatz physischer oder psychischer Gewalt ?Und wenn Du nicht willst, dann brauch? Gewalt!?

Stufe 2: Tauschangebot: ?Kratz? mir den Rücken, dann kratz? ich deinen!?

Stufe 3: Ausnutzung von Gruppensolidarität: ?Mein grosser Bruder/meine grosse Schwester wird mir beistehen!?

Stufe 4: Anrufung des Gesetzes: ?Ich bin im Recht!?

Stufe 5: Appell an gemeinsame Vertragsbasis: ?Du hast mir das aber versprochen!?

Stufe 6: Bezug auf universelle moralische Prinzipien: ?Das wäre die gerechteste Lösung für alle Beteiligten!?

Abbildung 1. Ebenen des Problemlösens (nach L. Kohlbergs Stufen der Moralentwicklung) 


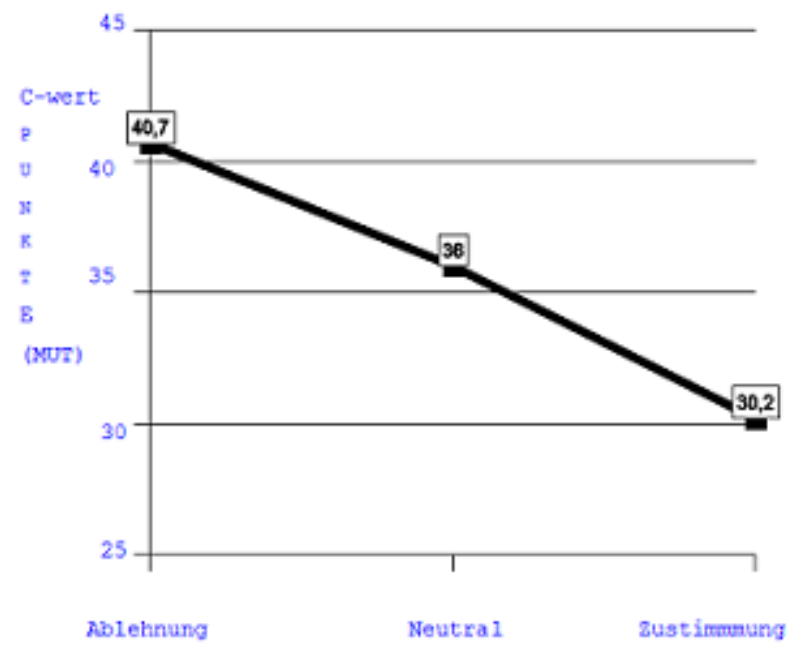

Abbildung 2. Änderung durch gewaltsame Störungen

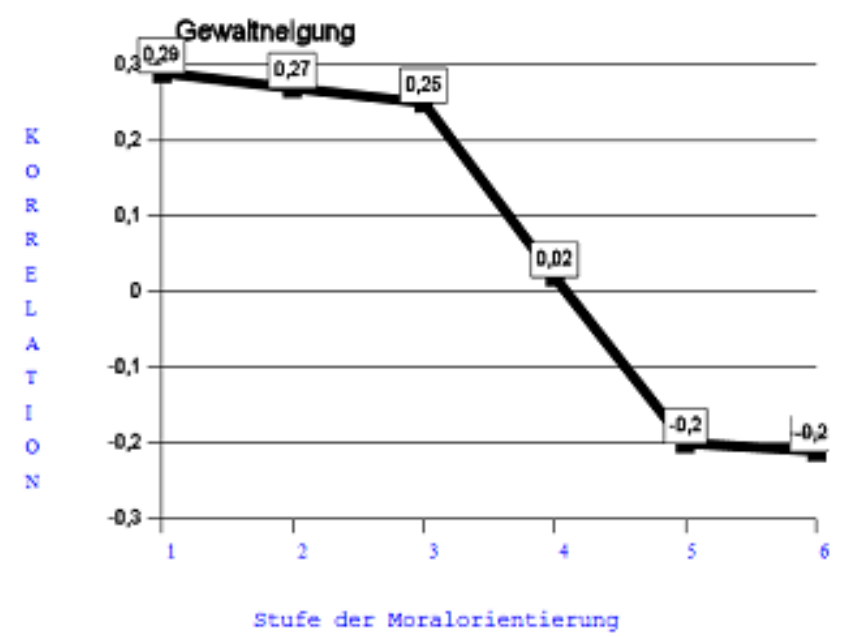

Abbildung 3. Gewaltneigung und moralische Orientierung

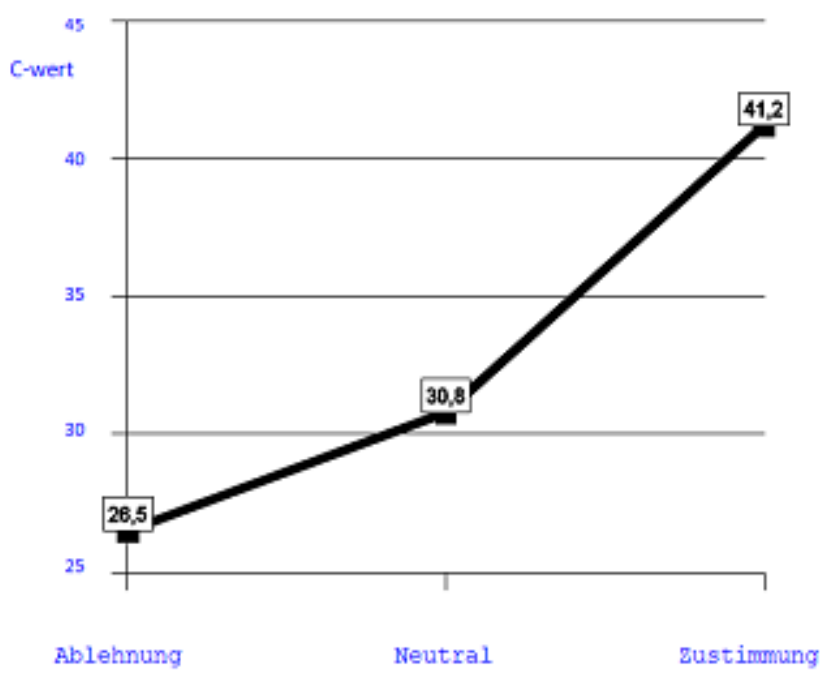

Abbildung 4. Demokratisierung aller Lebensbereiche 
* Originally published as: Lind, G. (1998). Gewalt und Krieg als niedrigste Stufe der Konfliktbewältigung. [Violence and war as the lowest stage of conflict resolution]. In: W. Kempf \& I. Schmidt-Regener, Eds., Krieg. Nationalismus, Rassismus und die Medien. [War. Nationalism, Racism and the Media]. Münster: LIT-Verlag, pp. 273-282.

1. Angemerkt sei, dass sich die Bedeutsamkeit dieser Unterschiede nicht, wie immer noch vielfach angenommen, aus statistischen Signifikanztests ablesen lässt, da diese uns nur etwas über statistische Bedeutsamkeit verraten, nicht aber über psychologische (Shaver,1993). (context) 